\title{
Randomized gap and amplitude estimation
}

\author{
Ilia Zintchenko ${ }^{1}$ and Nathan Wiebe ${ }^{1}$ \\ ${ }^{1}$ Quantum Architectures and Computation Group, Microsoft Research, Redmond, WA 98052, USA
}

\begin{abstract}
We provide a new method for estimating spectral gaps in low-dimensional systems. Unlike traditional phase estimation, our approach does not require ancillary qubits nor does it require well characterised gates. Instead, it only requires the ability to perform approximate Haar-random unitary operations, applying the unitary whose eigenspectrum is sought out and performing measurements in the computational basis. We discuss application of these ideas to in-place amplitude estimation and quantum device calibration.
\end{abstract}

PACS numbers: 03.67.Ac, 03.65.Wj, 03.67.Lx

\section{INTRODUCTION}

In recent years a host of methods have been developed for performing phase estimation in quantum systems [1 4]. These methods, driven by demand in quantum algorithms and metrology, have provided ever more efficient means of learning the eigenvalues of a quantum system. Through the use of sophisticated ideas from optimization and machine learning, recent phase estimation methods come close to saturating the Heisenberg bound [3] 6 , which is the ultimate performance limit for any phase estimation algorithm.

Despite the near-optimality of existing methods, there are a number of avenues of inquiry that still remain open surrounding phase estimation. Importantly, recent work has begun to look at operational restrictions on phase estimation including limited visibility in measurements, de-coherence and time-dependent drift. Such generalisations are especially important as we push towards building a scalable quantum computer and are faced with the challenge of characterising the gates in a massive quantum system that is imperfectly calibrated.

One important restriction that has not received as much attention is the issue that traditional phase estimation algorithms require entangling the quantum device with one (or more) ancilla qubits. This need to couple the system with an external qubit precludes ideas from phase estimation to be applied to single qubit devices. Even if ancilla qubits are available, such characterisation methods would entail the use of entangling gates which are very costly in many quantum computing platforms.

Our work provides a way to circumvent this problem for small quantum devices. It allows an experimentalist to learn the spectral gaps in an uncharacterised device with an amount of experimental time that is proportional to that of phase estimation without requiring any ancillary qubits. The idea of our approach is reminiscent of randomised benchmarking [7-10, and particularly iterated interleaved benchmarking [11], in that we use random operations to extract information about the underlying dynamics of the system.

We consider three applications for this method, which we call randomised gap estimation:

1. Eigenspectrum estimation for small quantum systems that lack well characterised gates.

2. Ancilla-free amplitude estimation.

3. Control map learning for small quantum devices.

This last application is interesting in the context of quantum bootstrapping [12 because it gives an inexpensive way of calibrating a set of one and two qubit gates at the beginning of a bootstrapping protocol. This is significant because an important caveat in bootstrapping is that while a trusted simulator can be used to quickly learn how to control an uncalibrated system, calibrating that trusted simulator requires exponentially more measurements and polynomially more experimental time than using traditional methods. Thus our techniques can, under some circumstances, dramatically reduce the total cost of a bootstrapping protocol.

Our paper is laid out as follows. We first provide a review of Bayesian inference in Section II and discuss the method for approximate Bayesian inference that we use in our numerical studies. We then introduce randomised gap estimation in Section III and discuss the challenges faced when applying the method to high-dimensional systems. We then show how the adiabatic theorem can be used to eliminate this curse of dimensionality for certain systems in Section IV] We then apply randomised gap estimation to the problem of amplitude estimation in $[$ Section V] and also to learning a map between experimental controls and the system Hamiltonian for a quantum device in Section VI] before concluding. 


\section{BAYESIAN INFERENCE}

Bayesian inference is a widely used method to extract information from a system. The goal of Bayesian inference is to compute the probability that a hypothesis $x$ is true given evidence $E$ and a set of prior beliefs about the hypotheses. These prior beliefs are represented as a probability distribution known as a prior. This prior, along with evidence $E$, can be thought of as an input to the inference algorithm. The output of the algorithm is the posterior distribution which is given by Bayes' theorem as

$$
P(x \mid E)=\frac{P(E \mid x) P(x)}{\int P(E \mid x) P(x) \mathrm{d} x},
$$

where $P(E \mid x)$ is known as the likelihood function. The posterior distribution output by this process is then used as a prior distribution in online inference algorithms and this process of generating a new prior distribution using Bayes' rule is known as a Bayesian update.

As an example of how Bayesian inference plays a role in eigenvalue estimation, consider the traditional approach used for iterative phase estimation. In iterative phase estimation, one wishes to learn the eigenvalues of a unitary operation $U$ eigenstates using the following circuit:

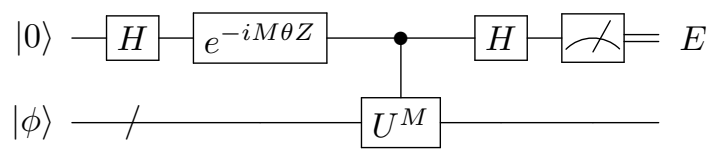

where $M$ and $\theta$ are user-specifiable experimental parameters, $H$ is the Hadamard matrix and $Z$ is the Pauli- $Z$ operator. Let $|\phi\rangle$ be an eigenstate such that $U|\phi\rangle=e^{i \lambda}|\phi\rangle$. The likelihood function for this circuit is then

$$
P(E=0 \mid \lambda ; \theta, M)=\cos ^{2}(M(\theta-\lambda))
$$

Note that in the above circuit we assume that $|\phi\rangle$ is an eigenstate. If it is not then the protocol will allow us to learn an eigenvalue corresponding to an eigenvector within the support of $|\phi\rangle[1]$.

The problem of phase estimation is thus the problem of estimating $\lambda$ given a sequence of different experimental outcomes. This can be done using (1) starting from an initial prior distribution for $\lambda$ that is uniform over $[0,2 \pi)$. A further benefit is that the posterior variance provides an estimate of the uncertainty in the inferred phase as well as an estimate of the most likely value of $\lambda$. This approach is considered in [2, 3] wherein exact Bayesian inference is found to perform extremely well both in settings where $\theta$ and $M$ are chosen adaptively as well as non-adaptively.

If $P(x)$ has support over an infinite number of points then exact Bayesian inference is usually intractable and discretisations are often employed to address this problem. Such discretisations include particle filter methods and sequential Monte Carlo methods [13 15. Here we employ a newly developed approach known as rejection filter inference [3, 16].

The idea behind Rejection filtering is to use rejection sampling to convert an ensemble of samples from the prior distribution to a smaller set of samples from the posterior distribution. Specifically, if evidence $E$ is observed and we draw a sample from the prior distribution and accept it with probability equal to $P(E \mid x)$ then the probability that hypothesis $x$ is accepted as a sample is from Bayes' theorem

$$
P(E \mid x) P(x) \propto P(x \mid E)
$$

Therefore, the samples that pass through the rejection filter are distributed according to the posterior distribution.

Although this process allows us to sample from the posterior distribution, it is not efficient. This is because every time we perform an update, we will, on average, reject a constant fraction of samples. This means that the number of samples kept will shrink exponentially with the number of updates. We can, however, make this process efficient by fitting these samples to a family of distributions and then draw a new set of samples from this model distribution for the next update. This ability to regenerate samples allows rejection filtering inference to avoid this catastrophic loss of support for the approximation to the posterior.

There are a number of models for the prior and posterior that can be considered. Here we use a unimodal Gaussian distribution. Gaussian models for the posterior distribution provide a number of advantages. First, they are parametrised by the posterior mean and covariance matrix which give an estimate of the true hypothesis and the uncertainty in it. Furthermore, these quantities are easy to estimate from the accepted samples and can be computed incrementally, which allows our algorithm to be executed using near-constant memory.

There are several advantages to using rejection filtering for approximate inference. Specifically, it is very fast, easy to parallelise, can be implemented using far less memory than particle filter or sequential Monte-Carlo methods. 


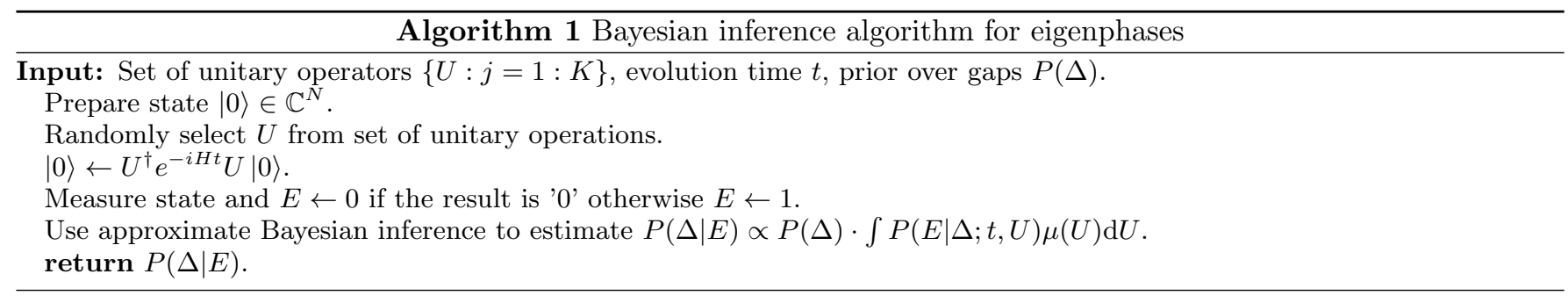

Perhaps most importantly, it is also substantially easier to implement than traditional particle filters. Rejection filtering has also been successfully used in phase estimation algorithms [3], where the use of rejection filtering leads to substantial reductions in the experimental time needed to perform the inference relative to Kitaev's phase estimation algorithm [1] and information theoretic phase estimation [2]

\section{RANDOMIZED GAP ESTIMATION}

Traditional approaches for learning the eigenspectrum of a Hamiltonian require ancillary qubits and well characterised gates. Here we present an approach, which we call randomised gap estimation, to efficiently estimate the eigenspectrum of a small system with no ancillary qubits and potentially poorly characterised gates. The idea behind this approach is to use Bayesian inference in concert with random evolutions to infer the gaps between the eigenphases of a unitary operator.

In the first step, the state

$$
|\Psi\rangle:=U|0\rangle=\sum_{k} \beta_{k}\left|v_{k}\right\rangle
$$

is prepared, where $\left|v_{k}\right\rangle$ is the eigenvector corresponding to eigenvalue $\lambda_{k}$ of $H$ in an arbitrary ordering. Here $\beta_{k}$ are unknown parameters that depend not only on the random unitary chosen, but also the eigenbasis of $H$. For low-dimensional systems exact methods for drawing $U$ uniformly according to the Haar measure are known (more generally it suffices to draw $U$ from a unitary 2-design see Section III A for more details). For example, a single qubit system $U$ has an Euler angle decomposition of

$$
U=R_{z}(\phi) R_{x}(\theta) R_{z}(\psi)
$$

up to an irrelevant global phase.

Next, we evolve the system according to $e^{-i H t}$ for a controlled time $t$. This results in the state

$$
e^{-i H t}|\Psi\rangle=e^{-i H t} U|0\rangle \text {. }
$$

Finally, $U^{\dagger}$ is applied and a measurement in the computational basis is performed, which returns '0' with probability

$$
\begin{aligned}
P(0 \mid H ; t, U) & :=\left|\left\langle 0\left|U^{\dagger} e^{-i H t} U\right| 0\right\rangle\right|^{2} \\
& =\left(\sum_{k}\left|\beta_{k}\right|^{2} \cos \left(\lambda_{k} t\right)\right)^{2}+\left(\sum_{k}\left|\beta_{k}\right|^{2} \sin \left(\lambda_{k} t\right)\right)^{2} \\
& =\sum_{i j} \cos \left(\left(\lambda_{i}-\lambda_{j}\right) t\right)\left|\beta_{i}\right|^{2}\left|\beta_{j}\right|^{2}:=P(0 \mid \Delta ; t, U) .
\end{aligned}
$$

Note that the gaps $\Delta_{i j}=\lambda_{i}-\lambda_{j}$ cannot be easily learned from this expression because of the unknown $\beta_{i}$ and $\beta_{j}$ terms. Haar averaging provides a solution to this problem.

Given an unknown Haar-random unitary $U$, the likelihood of measuring ' 0 ' is given by the law of conditional probability

$$
P(0 \mid \Delta ; t)=\int P(0 \mid \Delta ; t, U) \mu(U) \mathrm{d} U
$$

where $\mu$ is the Haar-measure over $U(N)$. This probability is distinct from the likelihood that would be used if the user knew, or was capable of computing, $P(0 \mid H ; t, U)$ for the particular $U$ that was used in the experiment. 
If we define $\Delta$ to be a matrix such that $\Delta_{i, j}:=\lambda_{i}-\lambda_{j}$, this Haar average evaluates to

$$
\begin{aligned}
P(0 \mid \Delta ; t): & =N\left\langle\left|\beta_{i}\right|^{4}\right\rangle+\left\langle\left|\beta_{i}\right|^{2}\left|\beta_{j}\right|^{2}\right\rangle_{i \neq j} \sum_{i \neq j} \cos \left(\Delta_{i j} t\right) \\
& =\frac{2}{N+1}\left(1+\frac{1}{N} \sum_{i>j} \cos \left(\Delta_{i j} t\right)\right) .
\end{aligned}
$$

Eq. (9) provides a likelihood function that can be used to perform Bayesian inference. In particular, if a binary experiment is performed wherein the only two outcomes are $|0\rangle$ and $|v \neq 0\rangle$, the latter occurs with probability $1-$ $P(0 \mid H ; t)$. If we define our prior distribution over eigenvalues to be $P(\lambda)$, then given a measurement of ' 0 ' is recorded, Bayes' rule states that the posterior distribution is

$$
P(\lambda \mid 0 ; t)=\frac{2\left(1+\frac{1}{N} \sum_{i>j} \cos \left(\Delta_{i j} t\right)\right) P(\lambda)}{(N+1) P(0)},
$$

where $P(0)$ is a normalisation factor. We outline this approach in Algorithm 1. Thus Bayesian inference allows the gaps to be learned from such experiments.

The Cramér-Rao bound provides an estimate for the minimum number of experiments and/or experimental time needed to ensure that the variance of $\Delta_{i j}$ is sufficiently small [17. If we assume that $R$ experiments are performed, each with $D \in O(1)$ outcomes and evolution time at least $t$, then the elements of the Fisher matrix are

$$
I_{i j, k l}=\sum_{d_{1}=0}^{D-1} \cdots \sum_{d_{R}=0}^{D-1} \frac{\partial_{\Delta_{i j}} \prod_{q=1}^{R} P\left(d_{q} \mid \Delta\right) \partial_{\Delta_{k l}} \prod_{q=1}^{R} P\left(d_{q} \mid \Delta\right)}{\prod_{q=1}^{R} P\left(d_{q} \mid \Delta\right)} \in O\left(\frac{R^{2} t^{2}}{N^{2}}\right),
$$

which through the use of the Cramér-Rao bound implies that the variance of any unbiased estimator of $\Delta_{i j}$ after all $R$ experiments scales at least as $\Omega\left(N^{2} / R^{2} t^{2}\right)$.

While this shows that the number of experiments needed to learn the gaps grows at least linearly with $N$, the uncertainty in the optimal unbiased estimator also shrinks as $\Omega(1 / T)$, where $T$ is the total evolution time. The error scaling is hence quadratically smaller than would be expected from statistical sampling. This opens up the possibility of very high precision frequency estimates for small quantum devices.

\section{A. Implementing Haar random unitaries}

In practice, Haar-random unitaries can be hard to exactly implement on a quantum computer [18. This task is even more challenging in an uncalibrated device. Fortunately, efficient methods for implementing pseudo-random unitaries are well known. Here we use the definition of $t$-design given by Dankert et al [7, wherein a unitary $t$-design on $N$ dimensions is taken to be a finite set of unitary operations such that the average of any polynomial function of degree at most $t$ in the matrix elements and their complex conjugates agrees with the Haar average. In particular, let $P_{(t, t)}\left(U_{k}\right)$ be such a polynomial function and assume that there are $K$ elements in this design. Then

$$
\frac{1}{K} \sum_{k=1}^{K} P_{(t, t)}\left(U_{k}\right)=\int P_{(t, t)}(U) \mu(U) \mathrm{d} U
$$

where $\mu$ is the Haar-measure.

More generally, one can also consider the notion of an $\epsilon$-approximate $t$-design. This notion differs from a $t$-design in that strict equality is not required and a discrepancy of at most $\epsilon$ in the diamond-distance is permitted. Specifically, let $G_{W}(\rho)=\sum_{i} U_{i}^{\otimes k} \rho\left(U_{i}^{\dagger}\right)^{\otimes k}$ be a super-operator corresponding to twirling the input operator over the elements of the design and $G_{H}(\rho)=\int U^{\otimes k} \rho\left(U^{\dagger}\right)^{\otimes k} \mu(U) \mathrm{d} U$ be the same quantity averaged over the Haar-measure. Then the set of unitary operators forms a $\epsilon$-approximate $k$-design if $\left\|G_{W}-G_{H}\right\|_{\diamond} \leq \epsilon$, where the diamond norm is discussed in detail in [19].

This definition implies that the Haar-expectations of the terms in (9) can be found using a 2-design since all such terms are quadratic in the matrix elements of $U$ and their complex conjugates. The remaining question is how to form such a design.

Dankert et al. show that random Clifford operations can be used to form exact and $\epsilon$-approximate 2-designs using $O\left(n^{2}\right)$ and $O(n \log (1 / \epsilon))$ gates respectively [7]. These results are sufficient in settings where the device has access 

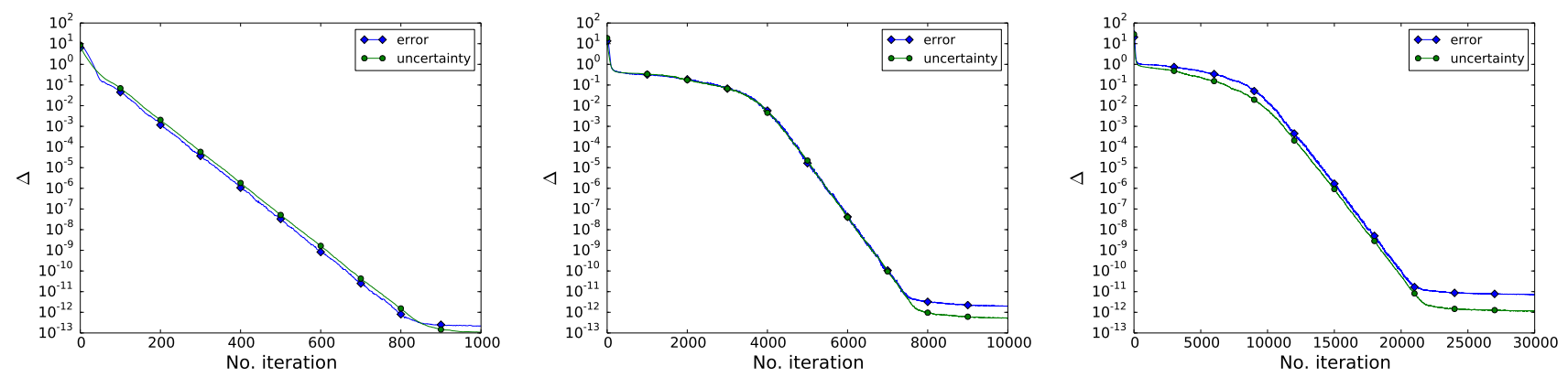

FIG. 1: Median error and uncertainty in the eigenvalues computed using randomised gap estimation using rejection filtering for 2-level (left), 3-level (middle) and 4-level (right) Hamiltonians with eigenspectra drawn from the Gaussian Unitary Ensemble.

to well characterised Clifford gates. If the system does not have such gates, the result of Harrow and Low 20] can be applied to show that $\epsilon$-approximate $t$-designs can be formed out of random sequences of gates taken from any universal gate set. The number of gates needed to generate such a pseudo-random unitary is $O(n(n+\log (1 / \epsilon)))[20$. in such cases, where the multiplicative constant depends on the details of the universal gate set used.

The result of Harrow and Low is especially significant here because the gates do not need to be known for it to hold (however we still require that the exact inverses of the uncalibrated gates can be performed). This means that even if we want to use randomised gap estimation to learn how to control certain uncalibrated quantum systems then we do not need to explicitly know the gates that are actually applied to the system to sufficiently randomise the measurement results if the underlying gate set is universal and a sufficiently long sequence of random operations is used.

\section{B. Numerical tests}

While the prior argument suggests that the error should scale as $1 / T$ for randomised gap estimation, an important question remains regarding how well it actually scales in practice. We assess this by using rejection filtering to estimate the gaps and the particle guess heuristic, discussed in [12, 21, 22 and also the appendix, to set the time for each experiment. We use this approach because it is fast, accurate and more importantly easier to implement than sequential Monte-Carlo methods [23, 24].

Although performing rejection filtering to infer the gaps may seem straight forward, a complication emerges that makes it conceptually more challenging to apply the technique directly. To see this, consider a Gaussian prior over the eigenvalue gaps for an $N$-dimensional system. If the eigenvalue gaps are drawn independently, the result will with high probability have inconsistent eigenvalue gaps. By inconsistent we mean that if $\Delta_{43}=\lambda_{4}-\lambda_{3}$ and $\Delta_{32}=\lambda_{3}-\lambda_{2}$ are eigenvalue gaps, then $\Delta_{42}=\lambda_{4}-\lambda_{2}=\Delta_{43}+\Delta_{32}$ must also be one of the eigenvalue gaps for the system. If $\Delta_{43}$ and $\Delta_{32}$ are chosen independently from a Gaussian prior, then it is very unlikely that their sum is also. Hence, gaps chose independently from the prior distribution will not correspond to a feasible set of eigenvalues.

There are many ways that this self-consistency constraint could be imposed in the inference step, but perhaps the simplest approach is to use Bayesian inference to learn a set of consistent eigenvalues for the system, rather than gaps directly. The solution is not unique 25] as we discuss further in Appendix B. However, any gap inferred from the potentially erroneous spectrum will be automatically consistent. We enforce this by taking the lowest-eigenvalue to be zero without loss of generality and then define the error in the inference as

$$
\Delta=\min \left\{\sum_{i}\left|\lambda_{i}-\mu_{i}\right|, \sum_{i}\left|\lambda_{i}-\left(\max \left\{\mu_{i}\right\}_{i}-\mu_{i}\right)\right|\right\}
$$

and the uncertainty as

$$
\sigma=\sqrt{\operatorname{Tr}(\Sigma)}
$$

given that rejection filtering reports a covariance matrix $\Sigma$ with a mean of $\mu_{i}$ for each eigenvalue $i$. The minimisation of the error in (13) is meant to remove the mirror symmetry that naturally arises as any sorted eigenspectrum gives the same distribution of gaps as its reflection about the midpoint (see Appendix B). We find numerically that Bayesian inference can rapidly estimate the eigenvalues of an unknown Hamiltonian, up to a degeneracy, in one and 
Algorithm 2 Bayesian inference algorithm for eigenphases

Input: Set of unitary operators $\{U: j=1: K\}$ such that $U=\mathbb{1} \otimes V_{j}$ where $V$ acts on a $M \leq N$ dimensional subspace of the computational basis, a diagonal Hamiltonian $H_{0}$, annealing time $T$, an interpolation function $f:[0,1] \mapsto[0,1]$ such that $f(0)=1-f(1)=0$, evolution time $t$ and prior over $M$ smallest gaps gaps $P(\Delta)$.

Prepare state $|0\rangle \in \mathbb{C}^{N}$.

Randomly select $U$ from set of unitary operations.

$|0\rangle \leftarrow U^{\dagger}\left(\mathcal{T} e^{-i \int_{0}^{1}(1-f(s)) H_{0}+f(s) H \mathrm{~d} s T}\right)^{\dagger} e^{-i H t}\left(\mathcal{T} e^{-i \int_{0}^{1}(1-f(s)) H_{0}+f(s) H \mathrm{~d} s T}\right) U|0\rangle$.

Measure state and $E \leftarrow 0$ if the result is ' 0 ' otherwise $E \leftarrow 1$.

Use approximate Bayesian inference to estimate $P(\Delta \mid E) \propto P(\Delta) \cdot \int P(E \mid \Delta ; t, U) \mu(U) \mathrm{d} U$.

return $P(\Delta \mid E)$.

two qubit systems (see Figure 1). In particular, we see that the error shrinks exponentially with the number of measurements before being ultimately limited by machine $-\epsilon$. This illustrates that randomised gap estimation can learn the eigenvalue gaps for low-dimensional systems using exponentially less data than would ordinarily be required by statistical sampling, although with only a quadratic advantage in the total experimental time.

For higher-dimensional systems achieving the same task is expected to be much more daunting and the error metric in (13) should be the minimum over all consistent re-orderings of the spectrum. Such problems can, however, be sidestepped in systems with a gapped adiabatic path connecting the Hamiltonian to a efficiently diagonalizable Hamiltonian, as we show below.

\section{ADIABATIC ELIMINATION OF EIGENVALUES}

Most eigenvalue estimation tasks in quantum computing focus on learning only a part of the spectrum rather than all the eigenvalues. We could use randomised gap estimation to learn the eigenvalues in this part of the spectrum if we could apply Haar-random unitary operations in only that subspace. Since the eigenvectors of the Hamiltonian are generally not known, it is difficult to do this directly. Despite this fact, the adiabatic theorem provides a means by which these random unitaries can be applied to the appropriate subspace by employing adiabatic state preparation.

Let us assume we wish to learn the spectrum, up to an additive constant, for a given subspace of a Hamiltonian $H_{p}$. In particular let $S=\operatorname{span}\left(\left|\lambda_{j_{1}}\right\rangle, \ldots,\left|\lambda_{j_{m}}\right\rangle\right)$ be a subspace of eigenvectors of $H_{p}$ such that the eigenvalues obey $\lambda_{1} \leq \lambda_{2} \leq \cdots$ and $j$ is a monotonically increasing sequence on $\left\{1, \ldots, 2^{n}\right\}$. Then we define an adiabatic interpolation to be a time-dependent Hamiltonian of the form $H(s)$ such that $H(0)=H_{0}$ and $H(1)=H_{p}$ where $H(s)$ is at least three-times differentiable for all $s \in(0,1)$ (where $s$ is the dimensionless evolution time) and furthermore has a gapped spectrum for all times in this interval. A common example of such a Hamiltonian is

$$
H(s)=(1-s) H_{0}+s H_{p} .
$$

The adiabatic theorem [26 28] shows that if $\left|\lambda_{j}^{0}\right\rangle$ is an eigenvector of $H_{0}$ whose eigenvalue corresponds to that of $\left|\lambda_{j}\right\rangle$ in the sorted list of eigenvalues,

$$
\mathcal{T} e^{-i \int_{0}^{1} H(s) \mathrm{d} s T}\left|\lambda_{j_{k}}^{0}\right\rangle=e^{i \omega_{j_{k}} T}\left|\lambda_{j_{k}}\right\rangle+O(1 / T),
$$

where $\mathcal{T}$ is the time-ordering operator. Hence, if we can perform a Haar-random unitary on the subspace of eigenvectors of $H_{0}$, denoted by $S_{0}=\operatorname{span}\left(\left|\lambda_{j_{1}}^{0}\right\rangle, \ldots,\left|\lambda_{j_{m}}^{0}\right\rangle\right)$, we can adiabatically transform the resultant state to a Haarrandom state in $S$, up to phases on each of the eigenvectors and error $O(1 / T)$.

Now let $U$ be a Haar-random unitary acting on $S_{0}$. Then the new adiabatic protocol for estimating the eigenphase is

$$
P(0 \mid H ; t, U)=\left|\left\langle 0\left|U^{\dagger}\left(\mathcal{T} e^{i \int_{1}^{0} H(s) \mathrm{d} s T}\right) e^{-i H_{p} t}\left(\mathcal{T} e^{-i \int_{0}^{1} H(s) \mathrm{d} s T}\right) U\right| 0\right\rangle\right|^{2}
$$

Given $U|0\rangle=\sum_{j:\left|\lambda_{j}\right\rangle \in S_{0}} \alpha_{j}\left|\lambda_{j}^{0}\right\rangle$ and $\tilde{U}|0\rangle:=\sum_{j:\left|\lambda_{j}\right\rangle \in S} \alpha_{j}\left|\lambda_{j}\right\rangle$, (16) shows that

$$
\left(\mathcal{T} e^{-i \int_{0}^{1} H(s) \mathrm{d} s T}\right) U|0\rangle=\sum_{j:\left|\lambda_{j}\right\rangle \in S_{0}} \alpha_{j} e^{i \omega_{j} T}\left|\lambda_{j}\right\rangle+O(1 / T) .
$$


Then using the fact that $H_{p}\left|\lambda_{j}\right\rangle=\lambda_{j}\left|\lambda_{j}\right\rangle$, we see from (17) and (18) that

$$
\begin{aligned}
P(0 \mid H ; t, U) & =\left(\sum_{k:\left|\lambda_{k}\right\rangle \in S}\left|\alpha_{k}\right|^{2} \cos \left(\lambda_{k} t\right)\right)^{2}+\left(\sum_{k:\left|\lambda_{k}\right\rangle \in S}\left|\alpha_{k}\right|^{2} \sin \left(\lambda_{k} t\right)\right)^{2}+O(1 / T) . \\
& =\left|\left\langle 0\left|\tilde{U}^{\dagger} e^{-i H_{p} t} \tilde{U}\right| 0\right\rangle\right|^{2}+O(1 / T) .
\end{aligned}
$$

The adiabatic theorem can therefore be used to allow randomised gap estimation to be performed on a specified subspace of eigenvalues if there exists a gapped adiabatic path between a Hamiltonian that is diagonal in the computational basis and the problem Hamiltonian (see Algorithm 2 for an outline of this approach). This shows that the curse of dimensionality that afflicts this method can be excised in cases where such an adiabatic evolution is possible. Randomized gap estimation can thus, in principle, be used as a fundamental tool to characterise and calibrate untrusted quantum systems. We will examine this further in subsequent sections.

As a final note, the asymptotic scaling of the error that emerges because of diabatic leakage out of the eigenstates, or more generally eigenspaces, can be exponentially improved using boundary cancellation methods [29 32], which set one or more derivatives of the Hamiltonian to zero at the beginning and end of the evolution. However, while this can substantially reduce the cost of the adiabatic transport, it requires fine control of the system Hamiltonian in order to realise the benefits of the cancellation [31. As a result, it is not necessarily clear when the higher-order versions of these algorithms will find use in applications outside of fault tolerant quantum computing.

\section{APPLICATION TO AMPLITUDE ESTIMATION}

Randomised gap estimation also provides an important simplification for amplitude estimation, which is a quantum algorithm in which the probability of an outcome occurring is estimated by combining ideas from amplitude amplification and phase estimation [33. The algorithm quadratically reduces the number of queries needed to learn a given probability. The main significance of this is that it quadratically speeds up Monte-Carlo algorithms. Specifically, imagine that you are given an unknown quantum state of the form

$$
|\psi\rangle:=A|0\rangle=a|\phi\rangle+\sqrt{1-|a|^{2}}\left|\phi^{\perp}\right\rangle,
$$

where $|\phi\rangle \in \mathbb{C}^{N}, A$ is a unitary operator and $|a| \in(0,1)$. Furthermore, assume that you are given access to an oracle such that $\chi|\phi\rangle=-|\phi\rangle$ and for all states $|v\rangle$ orthogonal to $|\phi\rangle, \chi|v\rangle=|v\rangle$. Amplitude estimation then allows $|a|^{2}$ to be estimated to within error $\epsilon$ using $\tilde{O}(1 / \epsilon)$ applications of $\chi$ and $O(\log (1 / \epsilon))$ measurements.

In order to understand how this works, consider the Grover search operator $Q=-A \chi_{0} A^{\dagger} \chi$, where $\chi_{0}$ acts as $\chi$ would for $\phi=0$. For the case of a single marked state we then have that

$$
Q^{j}|\psi\rangle=\sin \left([2 j+1] \theta_{a}\right)|\phi\rangle+\cos \left([2 j+1] \theta_{a}\right)\left|\phi^{\perp}\right\rangle,
$$

where $\theta_{a}:=\sin ^{-1}(a)$. It is then clear that $Q$ enacts a rotation in this two-dimensional subspace and has eigenvectors

$$
\left|\psi_{ \pm}\right\rangle=\frac{1}{\sqrt{2}}\left(|\phi\rangle \pm i\left|\phi^{\perp}\right\rangle\right)
$$

where

$$
Q\left|\psi_{ \pm}\right\rangle=e^{ \pm i 2 \theta_{a}}\left|\psi_{ \pm}\right\rangle
$$

All other eigenvectors in the space orthogonal to this have eigenvalue \pm 1 .

We can learn these phases using randomised gap estimation. The most significant change is that here $t$ must be taken to be an integer since fractional applications of the Grover oracle have not been assumed. In this case, the eigenvalues are in the set $\left\{-2 \theta_{a}, 0,2 \theta_{a}, \pi\right\}$, which implies that $\left|\Delta_{i, j}\right|$ takes the values $\left\{0, \pm 2 \theta_{a}, 4 \theta_{a}, \pi \pm 2 \theta_{a}, \pi\right\}$. This means that the gap estimation process has very different likelihoods if $t$ is even or odd. Since $\cos \left(\left[\pi \pm 2 \theta_{a}\right](2 p+1)\right)=-\cos \left(2 \theta_{a}(2 p+1)\right)$, for integer $p$, many of the terms in (9)] cancel if $t$ is odd. As a result, it is better to choose $t$ to be an even integer for this application, in which case the likelihood function is

$$
P\left(0 \mid \theta_{a} ; t\right)=\frac{2}{N+1}\left(1+\frac{1}{N}\left(\left(\begin{array}{c}
N-2 \\
2
\end{array}\right)+2(N-2) \cos \left(2 \theta_{a} t\right)+\cos \left(4 \theta_{a} t\right)\right)\right) .
$$




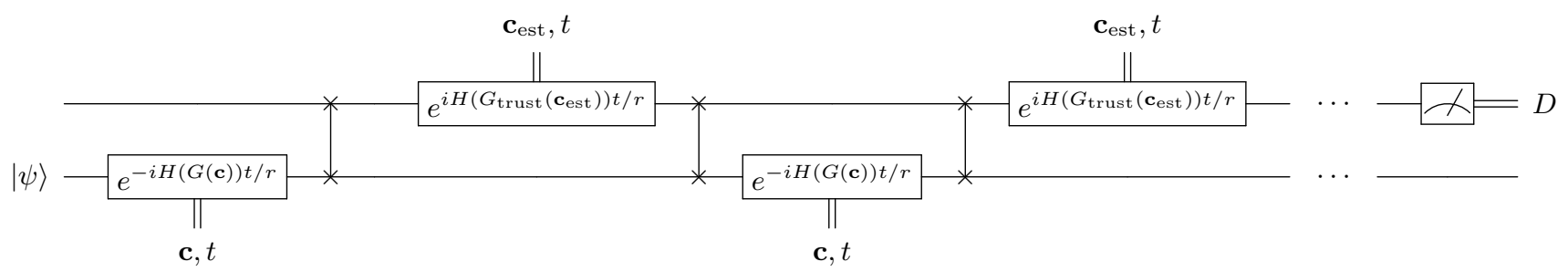

FIG. 2: Quantum bootstrapping experiment to learn a control map using interactions between a trusted simulator and an uncharacterised device. The value $r$ can be increased to combat the spread of non-local correlations and also to reduce ambiguities that can arise in the inference process when the untrusted and trusted Hamiltonians do not commute.

Such experiments cannot yield substantial information as $N \rightarrow \infty$ according to (11) and thus different ideas are needed to make large $N$ instances tractable unless we can sample according to the Haar measure only within the relevant subspace using techniques similar to those in Section IV]

This method is, however, viable without modification for small $N$. To illustrate this, consider the case where $N=2$, where the likelihood function reduces to

$$
P\left(0 \mid \theta_{a} ; t\right)=\frac{2}{3}\left(\frac{1}{2}+\cos ^{2}\left(2 \theta_{a} t\right)\right)
$$

This likelihood function yields only slightly less information than the one for iterative phase estimation, $P\left(0 \mid \theta_{a} ; t\right)=$ $\cos ^{2}\left(2 \theta_{a} t\right)$, as their derivatives with respect to $\theta_{a}$ differ by at most a constant factor. This observation, coupled with the fact that an additional qubit is not needed, means that amplitude estimation can be realistically applied in-place in single qubit devices using this method.

\section{APPLICATION TO CONTROL MAP LEARNING}

Another important application of randomised gap estimation is calibration of quantum devices. In order to understand why calibration can be a problem, imagine that you wish to calibrate a qubit so that you can perform an $X$ gate within error $\epsilon=10^{-5}$. In order to verify, using with plain statistical sampling, that the error is this small, roughly $10^{10}$ experiments are needed. This process would also have to be repeated for every quantum bit in the system, which could easily result in terabytes of data needed to calibrate a small fault tolerant quantum device. Our approach provides a method that can exponentially reduce the number of measurements needed to control such a qubit under these circumstances and polynomially reduce the experimental time needed.

In order to abstract the problem of calibrating an unknown quantum device, let us consider the problem of learning a control map. We define the control map to be the mapping between a set of experimental controls (specified by the vector $\mathbf{c}$ ) and the system Hamiltonian. Specifically, if we define $H(\mathbf{x})=\sum_{i=1}^{N} x_{i} H_{i}$ for a set of Hamiltonians $H_{i}$, then $\mathbf{x}$ is given by $\mathbf{c}$ via

$$
\mathbf{x}=G \mathbf{c}+\mathbf{x}_{0},
$$

where the matrix $G$ is known as the control map. For simplicity we will only consider the linear case where $\mathbf{x}_{0}=0$ below. The general case is handled by first learning $\mathbf{x}_{0}$ and is discussed in [12].

Recently, a method called quantum bootstrapping has been proposed to learn such maps efficiently, wherein a trusted quantum simulator is used to infer the controls of an untrusted device (see Figure 2 for a circuit diagram of a bootstrapping experiment). Although this can allow the control map to be learned using a poly-logarithmic number of experiments, it necessitates the use of swap gates that couple the device to the trusted simulator that is used to characterise it. This means that verifying that the trusted simulator is properly working can still require an exponential number of measurements (even under assumptions of locality). This is significant because the error in the trusted simulator places a lower limit on the error that can be attained using the experiments proposed in [12, 21 23]. Figure 3 demonstrates the dependence of the error in the inference of a control map for a single qubit that arises from the use of a miscalibrated trusted simulator that has $G$ drawn from a zero-mean Gaussian with covariance matrix $\delta \mathbb{1}$, where $\delta$ is chosen to take values within the interval $\left[0,10^{-2}\right]$. We see there that the error decays until it saturates at a level dictated by the miscalibrations in the trusted Hamiltonian. This illustrates that errors in the trusted simulator can prevent a bootstrapping protocol from perfectly controlling this idealised quantum system. 


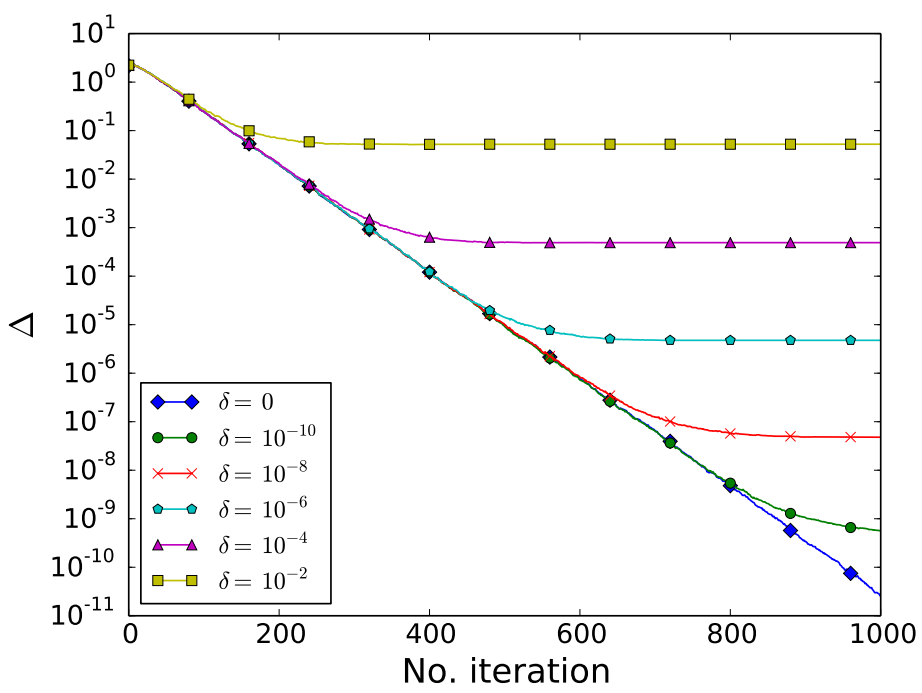

FIG. 3: Median error for a quantum bootstrapping protocol that attempts to learn a control map for a universal qubit using a miscalibrated second qubit to control the measurement basis with miscalibrations of size $\delta$. These results are simulated in the limit as $r \rightarrow \infty$ (see Figure 2) as rejection sampling filtering has poor success probability for non-commuting models because of (intermediate multi-modality), unlike the sequential Monte-Carlo methods with $r=1$ studied in [22].

It is worth noting that although Bayesian inference does not correctly infer the true control map for the system because of these errors in the trusted simulator, it does infer a set of controls that precisely mimics the trusted device. In this sense, the learning task can be thought of as a machine learning task, wherein the untrusted device is trained to maximise the fidelity of its output with that of the "trusted" simulator, wherein the output state can be thought of as the training data for the problem. It is therefore crucial to have a well-calibrated trusted device if we wish to leverage the full power of quantum bootstrapping.

To this end we provide a number of examples below that show how the control maps of single qubit Hamiltonians can be learned using randomised gap estimation. We thereby show that our techniques may be of great value in building a trusted simulator that can be used in bootstrapping protocols.

\section{A. Two level example}

In the case for a two-level, single-qubit, system, the Hamiltonian can be written, up to an irrelevant shift in energy, as

$$
H([\alpha, \beta, \gamma])=\alpha X+\beta Y+\gamma Z .
$$

Since these three operations anti-commute it is evident that the eigenvalues of $H$ are

$$
E= \pm \sqrt{\alpha^{2}+\beta^{2}+\gamma^{2}} .
$$

Thus we can infer information about $\alpha, \beta$ and $\gamma$, and in turn $G$, from the eigenspectra of different experiments. Specifically,

$$
\left[\begin{array}{l}
\alpha \\
\beta \\
\gamma
\end{array}\right]=\left[\begin{array}{lll}
G_{00} & G_{01} & G_{02} \\
G_{10} & G_{11} & G_{12} \\
G_{20} & G_{21} & G_{22}
\end{array}\right]\left[\begin{array}{l}
c_{1} \\
c_{2} \\
c_{3}
\end{array}\right] .
$$

The simplest example of control map learning is the diagonal case, for which

$$
E= \pm \sqrt{G_{00}^{2} c_{1}^{2}+G_{11}^{2} c_{2}^{2}+G_{22}^{2} c_{3}^{2}} .
$$

and the control map can be learned, up to signs of $G_{i j}$, using a sequence of three randomised phase estimation experiments with $c=[1,0,0],[0,1,0]$ and $[0,0,1]$. The signs can be inexpensively learned using auxiliary experiments 
because only a single bit of information is required. Thus empirically the number of experiments required to learn $G$ using randomised phase estimation is upper bounded by a constant times $\log (1 / \epsilon)$ (see Figure 1 ).

The next simplest case to consider is that of an upper triangular control map with a positive diagonal in the form

$$
\left[\begin{array}{l}
\alpha \\
\beta \\
\gamma
\end{array}\right]=\left[\begin{array}{ccc}
G_{00} & G_{01} & G_{02} \\
0 & G_{11} & G_{12} \\
0 & 0 & G_{22}
\end{array}\right]\left[\begin{array}{l}
c_{1} \\
c_{2} \\
c_{3}
\end{array}\right] .
$$

Clearly $G_{00}$ can be learned directly with randomised gap estimation using the prior experiments. However, the remaining elements must be inferred from different different experiments. If $c=[1,1,0]$ then

$$
\begin{aligned}
& E([1,1,0])= \pm \sqrt{\left(G_{00}+G_{01}\right)^{2}+G_{11}^{2}} \\
& E([0,1,0])= \pm \sqrt{G_{01}^{2}+G_{11}^{2}} .
\end{aligned}
$$

After squaring and subtracting both equations we find

$$
E^{2}([1,1,0])-E^{2}([0,1,0])=G_{00}^{2}+2 G_{00} G_{01},
$$

which can be solved uniquely for $G_{01}$ since $G_{00}$ is known. Once $G_{01}$ is known, $G_{11}$ can be learned unambiguously from (33) using the fact that $G_{11} \geq 0$.

After these steps we have inferred the first two columns of $G$. The remaining column can be learned similarly by performing three randomised gap estimation experiments with $c=[0,0,1],[1,0,1]$ and $[0,1,1]$ which yield

$$
\begin{aligned}
& E([0,1,1])= \pm \sqrt{\left(G_{01}+G_{02}\right)^{2}+\left(G_{11}+G_{12}\right)^{2}+G_{22}^{2}} \\
& E([1,0,1])= \pm \sqrt{\left(G_{00}+G_{02}\right)^{2}+G_{12}^{2}+G_{22}^{2}} \\
& E([0,0,1])= \pm \sqrt{G_{02}^{2}+G_{12}^{2}+G_{22}^{2}}
\end{aligned}
$$

Then by subtracting the square of (37) from (36) we learn $G_{02}$, from which we learn $G_{12}$ from substituting the result into (35) $G_{22}$ can then be learned by subtracting the square of (36) from (35) substituting the value of $G_{02}$ and using $G_{22} \geq 0$.

More generally, this approach provides information about the inner product between any two columns of $G$. No more information about $G$ can be extracted with such measurements. That is, $G^{T} G$ can be learned, but $G$ itself is only determined up to an orthogonal transformation $G^{\prime}=Q G$ which preserves the inner products of its columns. The matrix $Q$ can further be determined only if additional constraints are imposed on $G$. For example, if $G$ is upper or lower triangular and with a positive diagonal it is unique within such measurements and $Q$ is the identity matrix as discussed above.

\section{B. Learning $2 \times 2$ single qubit control maps}

As mentioned above, gap estimation experiments alone are in general not enough to uniquely specify the control map. In fact, amplitudes of the states in the eigenbasis are required. This raises the question of whether amplitude estimation may be used to glean the necessary information from these coefficients using $O(\log (1 / \epsilon))$ measurements.

For simplicity lets consider the Hamiltonian of a single qubit with only $X$ and $Z$ rotations

$$
H([\alpha, \gamma])=\alpha X+\gamma Z
$$

and

$$
\left[\begin{array}{l}
\alpha \\
\gamma
\end{array}\right]=\left[\begin{array}{ll}
G_{00} & G_{01} \\
G_{10} & G_{11}
\end{array}\right]\left[\begin{array}{l}
c_{1} \\
c_{2}
\end{array}\right]
$$

The case of a full $3 \times 3$ control map is similar, but we do not discuss it here because it is much more cumbersome owing to the increased number of equations and unknowns. 
Randomized gap estimation can provide

$$
\begin{aligned}
& E([1,0])= \pm \sqrt{G_{00}^{2}+G_{10}^{2}} \\
& E([0,1])= \pm \sqrt{G_{01}^{2}+G_{11}^{2}} \\
& E([1,1])= \pm \sqrt{\left(G_{00}+G_{01}\right)^{2}+\left(G_{10}+G_{11}\right)^{2}},
\end{aligned}
$$

Since there are four unknowns and three equations, the control map cannot be unambiguously learned from these relations. However, it can be seen that any other such randomised gap estimation provides information that is not linearly independent of these three equation. We need additional information that cannot directly come from randomised gap estimation to solve the problem. This problem was avoided in the prior example by constraining $G_{10}$ to be zero.

We can learn the required final piece of information by measuring expectation values of the following three operators

$$
\begin{aligned}
& A([1,0])=e^{-i\left(G_{00} X+G_{10} Z\right) \pi /(2 E([1,0]))}, \\
& A([0,1])=e^{-i\left(G_{01} X+G_{11} Z\right) \pi /(2 E([0,1]))}, \\
& A([1,1])=e^{-i\left(\left(G_{00}+G_{01}\right) X+\left(G_{10}+G_{11}\right) Z\right) \pi /(2 E([1,1]))} .
\end{aligned}
$$

These each correspond to free evolution of the system Hamiltonian under particular control settings for fixed durations. We can then use these operators (see eq $(20)$ to learn the following amplitudes while marking the $|0\rangle$ state

$$
\begin{aligned}
& |a([1,0])|=|\langle 0|A([1,0])| \mid 0\rangle|=\left|\frac{G_{10}}{E([1,0])}\right|, \\
& |a([0,1])|=|\langle 0|A([0,1])| 0\rangle|=\left|\frac{G_{11}}{E([0,1])}\right|, \\
& |a([1,1])|=|\langle 0|A([1,1])| \mid 0\rangle|=\left|\frac{G_{10}+G_{11}}{E([1,1])}\right| .
\end{aligned}
$$

If the sign of $G_{11}$ or $G_{10}$ is known, these three quantities are enough to unambiguously solve for $G_{11}$ and $G_{10}$, and can all be learned in-place using randomised amplitude estimation if an accurate $Z$-gate can be performed. The remaining elements of the control map can then be found from (40) If the sign of $G_{11}$ or $G_{10}$ is not known, they can still be learned with amplitude estimation if the states $(|0\rangle \pm i|1\rangle) / \sqrt{2}$ can be prepared on the quantum device.

In the absence of a calibrated $Z$ gate, the above strategy of amplitude estimation does not apply. The requisite quantities $|a([0,1])|,|a([1,0])|$ and $|a([1,1])|$ can nonetheless be learned by statistical sampling. Doing so requires $O\left(1 / \epsilon^{2}\right)$ experiments, which dominates the cost of data acquisition. However, randomised gap estimation efficiently provides $E([0,1]), E([1,0])$ and $E([1,1])$ which allows us to optimally extract these expectation values (for the form of the experiments considered). To see this consider the case of learning $|a([0,1])|$ for arbitrary $t$. The likelihood function is

$$
P(0 \mid G ; t)=\cos ^{2}(E([0,1]) t)+\sin ^{2}(E([0,1]) t)|a([0,1])|^{2} .
$$

It is then clear that the Fisher information is maximised at $t=\pi /(2 E([0,1]))$. In order to compare, let us consider the case where $E([0,1]) t \bmod 2 \pi$ is taken from a uniform prior over $0,2 \pi$. Then the marginalised likelihood is

$$
\frac{1}{2 \pi} \int_{0}^{2 \pi} \mathrm{d} x \cos ^{2}(x)+\sin ^{2}(x)|a([0,1])|^{2}=\frac{|a([1,0])|^{2}+1}{2} .
$$

thus (11) shows that the Fisher information for such experiments is reduced by a factor of 2 . This furthermore reduces the inference problem to frequency estimation wherein the non-adaptive strategy of estimating $|a([0,1])|^{2}$ using the sample frequency is optimal. Thus, even though randomised gap estimation does not lead to a quadratic reduction in the experimental time needed to learn $G$ here, it dramatically simplifies the inference problem and removes the need to use multiple experimental settings to learn the amplitudes.

Above we only discussed the case of a single qubit Hamiltonian with uncalibrated $X$ and $Z$ interactions. The case of a general (traceless) single qubit Hamiltonian follows from exactly the same reasoning. First, one applies randomised gap estimation to learn the relevant energies corresponding to the 6 linearly independent sets of controls. The remaining 3 equations are found by measuring appropriate expectation values using amplitude estimation if a $Z$ gate is available, and statistical sampling if it is not.

Finally, higher dimensional control maps can also be learned using this approach. The analytic approach given above no longer holds unless the Hamiltonian can be decomposed into a set of anti-commuting Pauli operators. The same process can, however, be applied numerically in such cases. We leave a detailed discussion of the issues that arise when doing so for future work. 


\section{CONCLUSION}

We introduced an alternative form of phase estimation that uses Bayesian inference on top of randomised experiments to learn the spectral gaps of a unitary operation without needing external qubits or well-calibrated gates. In addition to calibration, this gap estimation procedure allows amplitude estimation to be performed in place for quantum systems without needing auxiliary qubits to control the evolution. These randomised gap and amplitude estimation algorithms could radically reduce the costs of characterising and calibrating small quantum devices. Our work further suggests that they may find use in building the initial trusted simulator that is required to start a quantum bootstrapping protocol [12] as well as allowing amplitude estimation to be done in place in small quantum systems.

An important caveat of our work is that the randomisation procedure that we use to extract information about the gaps leads to an exponential decrease in the signal yielded by experiments on high-dimensional systems. We show that this can be mitigated, for some systems, using adiabatic paths to allow the randomisation to be performed only within a low-dimensional eigenspace of the system. Regardless, this caveat still holds in general and as such our methods are complimentary to existing phase and amplitude estimation methods and do not replace them.

This suggests an important remaining question namely that of whether efficient methods exist for estimating phase and amplitude that do not require entangling the system with ancillary qubits. The development of such methods would constitute a major step forward for quantum metrology. Similarly, proof that such methods are impossible would give us new insights into the efficacy of phase estimation and reveal fundamental trade offs that exist between quantum resources for metrological tasks.

Finally, it should be noted that our work simply uses information from the Haar averaged probability distribution to infer the eigenvalue gaps. In practice, additional information may be present in the higher order moments of the distribution. Finding practical ways to exploit information carried by these higher moments about the phase remains an open problem.

\section{Acknowledgments}

We thank Matt Hastings and Shawn Cui for valuable comments and introducing us to the turnpike problem as well as Michael Beverland, Alex Kubica, Christopher Granade and Paolo Zanardi for providing useful feedback on this work.

\section{Appendix A: Numerics}

Here we provide detailed information about the numerical experiments performed to generate Figure 1 . As mentioned in the main body we use sets of eigenvalues as our hypotheses for Bayesian inference. Without loss of generality we can shift the eigenspectrum such that $\lambda_{1}=0$ and we hence have $N-1$ variables, where $N$ is the number of levels.

For the Bayesian inference step, we used rejection filtering with a threshold of 10000 accepted samples. We found that drawing parameters from the model until 10000 samples are accepted provides sufficient accuracy to represent the likelihood function in these experiments. We use a standard unimodal multivariate Gaussian as a model for the prior distribution on hypothesis space. While this unimodal distribution is incapable of representing a multi-modal distribution (which may occur in cases where degenerate solutions exist), multimodal models can also be used to improve learning. However, for our experiments the mean and covariance of all modes quickly converged to the same value and the accuracy was hence no better than in the unimodal case.

The initial prior distribution is taken to be a Gaussian with mean and standard deviation set to be those appropriate for Gaussian Unitary Ensemble (GUE) Hamiltonians. In particular, we find these values using the gap distribution of GUE Hamiltonians and set the mean eigenvalue to be that found by adding $N-1$ random gaps drawn from the distribution appropriate for GUE Hamiltonians to $\lambda_{1}=0$. Similarly the covariance matrix for the $N-1$ unknown eigenvalues was set to be variances equivalent to those found for each of the eigenvalues of GUE Hamiltonians. While this distribution does not accurately model the true prior distribution of the eigenvalues, it has sufficient overlap to be able to zero in on the correct eigenvalues (up to degeneracies that cannot be resolved by randomised gap estimation alone).

Evolution times are chosen to be $t=1 /(2 \sigma)$, where $\sigma$ is the uncertainty defined in (14). This heuristic was introduced in previous works [12, 21, 22] as the particle guess heuristic and is shown to be nearly optimal for frequency estimation problems. The prefactor of $1 / 2$ was found to work well empirically, but more optimal choices may exist for this problem. 
Since degeneracies in the eigenvalues can emerge in our problem that prevent correct inference of the gaps, we resort to modifying the likelihood function in order to forbid particular Hamiltonian models. Specifically, we force the eigenvalues to be sorted and positive by introducing an additional sign-term in the likelihood function

$$
\langle L\rangle=\frac{2}{N+1}\left(1+\frac{1}{N} \sum_{i>j} \operatorname{sign}\left(\Delta_{i j}\right) \cos \left(\Delta_{i j} t\right)\right)
$$

where the sign-function is +1 if its argument is positive and -1 otherwise and $\Delta_{i j}=\lambda_{i}-\lambda_{j}$. Hence, this likelihood will align with the experimental likelihood at all time-steps $t$ only if $\lambda_{i}>\lambda_{j}$. As this modified likelihood can for some sets of parameters be larger than unity or smaller than zero, we do an additional truncation step such that $0 \leq L \leq 1$.

The median error and uncertainty reported are computed across 1000 instances drawn from the Gaussian Unitary Ensemble. This number was sufficient to make the sample error in the estimates graphically insignificant.

\section{Appendix B: The turnpike problem for unique gaps}

Eigenvalues are not directly provided in randomised phase estimation. Instead the eigenvalues for the system must be inferred from the gaps. In the main body, as well as the numerical experiments, we use Bayesian inference to infer the eigenvalues from the data. However, it remains an open question under what circumstances it can successfully infer the eigenvalues, up to a constant shift, from the gaps. The problem of inferring a set of numbers given only the differences between them is well known in computer science and is called the turnpike problem [25. The turnpike problem has proven difficult to solve in general and the best algorithm to find a solution remains an open problem. However, for the typical case, wherein the gaps are unique, there are at most two degenerate choices of spectra that could equivalently describe the system, as we show below.

Theorem 1. Let $\left\{\lambda_{1}:=0<\lambda_{2}<\cdots<\lambda_{N}\right\}$ be a set such that for any $i \neq k\left(\lambda_{i+1}-\lambda_{i}\right) \neq\left(\lambda_{k+1}-\lambda_{k}\right)$. Given $\Delta: \Delta_{x y}=\lambda_{p_{x}}-\lambda_{q_{y}}$ for unknown permutations of $\{1, \ldots, N\}$ and $q$, there are only two possible solutions for the values of $\lambda$.

Proof. The nearest neighbour gaps can be isolated from this set easily. Consider $\lambda_{i+2}-\lambda_{i}=\lambda_{i+2}-\lambda_{i+1}+\lambda_{i+1}-\lambda_{j}$. Since $\lambda_{i+2}-\lambda_{i+1}$ and $\lambda_{i+1}-\lambda_{i}$ are in $\Delta$ it follows that such a gap can be decomposed into a sum of gaps in the set. Similarly, by induction $\lambda_{i+x}-\lambda_{i}$ can be decomposed into a sum of nearest neighbour gaps for all $x \geq 2$. In contrast, the nearest neighbour difference $\lambda_{i+2}-\lambda_{i+1}$ cannot be decomposed into a sum of other gaps because of our assumptions of uniqueness. This means that $\Delta^{1}$, the set of all nearest neighbour gaps, can be uniquely identified from this data.

It remains to show that there are only two ways that these gaps can be laid out to form a spectrum that is consistent with the data in $\Delta$. The quantity $\Delta_{x y} \lambda_{N}$ can be identified from the spectrum since it is simply the largest gap under the assumption that $\lambda_{1}=0$. The second largest gap can also be uniquely found from this data. There are two possibilities. Either the second largest gap is $\lambda_{N-1}$ or it is $\lambda_{N}-\lambda_{2}$. This can be seen by contradiction. Imagine $\lambda_{N}-\lambda_{j}$ is the second largest gap for $j \geq 3$. Since $\lambda_{j}$ is a monotonically increasing sequence, $\lambda_{N}-\lambda_{j} \leq \lambda_{N}-\lambda_{2} \leq \lambda_{N}$. Therefore, it cannot be the second largest gap. Applying the same argument to $\lambda_{N-1}-\lambda_{j}$ for $j \geq 2$ leads to the conclusion that $\lambda_{N-1}$ is the only other candidate for the second largest gap. Similarly $\lambda_{x}-\lambda_{j} \leq \lambda_{N-1}-\lambda_{j}$ for all $x \leq N-1$ so these are the only two possible candidates.

Assume that the second largest gap is $\lambda_{N-1}$. Then

$$
\lambda_{N-2}=\left(\lambda_{N-2}-\lambda_{N-1}\right)+\lambda_{N-1} .
$$

Seeking a contradiction, imagine there is an alternative solution to this such that

$$
\lambda_{p}-\lambda_{N-1}=\left(\lambda_{r-1}-\lambda_{r}\right)
$$

If $\lambda_{p}-\lambda_{N-1}$ is not a nearest-neighbour gap, we have a contradiction, since the set of nearest-neighbour gaps is known and each gap is unique. Hence, we can immediately deduce that $\lambda_{p}$ is not a solution unless $p \in\{N-2, N\}$. Given $\lambda_{p}-\lambda_{N-1}$ is a nearest neighbour gap we must have that $p=N-2$ or $p=N$. Since the nearest neighbour gaps are unique, $\lambda_{N-2}-\lambda_{N-1} \neq \lambda_{N-1}-\lambda_{N}$ and since we already know $\lambda_{N}-\lambda_{N-1}$ this confusion is impossible. Furthermore, the uniqueness of the nearest neighbour gaps imply that the only possible solution is $r=N-1$. Thus $\lambda_{N-2}$ is uniquely determined.

This process can be repeated using $\lambda_{p}$ and $\lambda_{p+1}$ for any $P<N$. Therefore, given the second largest gap is $\lambda_{N-1}$, the spectrum can be uniquely determined under our assumptions. 
Now let us assume the second largest gap is $\lambda_{N}-\lambda_{2}$. Then $\lambda_{2}$ is known because $\lambda_{N}$ is the largest gap, given $\lambda_{1}=0$. By repeating the exact same argument as above we see that $\lambda_{3}$ is uniquely determined by these two values and the uniqueness of the nearest neighbour gaps. This same argument can be extended leading to the conclusion that if $\lambda_{2}$ is $\lambda_{N}$ minus the second largest gap then the spectrum is also unique. There are hence at most two possible spectra based on this data.

This approach shows that if the gaps are unique, a solution to the turnpike problem is also unique up to a reflection of the spectrum about its midpoint. However, in the presence of degenerate gaps the number of possible solutions could scale slightly faster than linear in $N$ 25] and the eigenspectrum can in this case only narrowed down to be one of these solutions. Moreover, while a backtracking algorithm can find a solution for the typical case in time $O\left(N^{2} \log (N)\right)$, the worst case complexity is $O\left(2^{N} N \log (N)\right)$ [25. This suggests that Bayesian inference will not always find a solution in time that is polynomial in $N$, which itself is exponential in the number of qubits. However, for the two level spectrum of a single qubit, the phase can be determined unambiguously.

[1] Alexei Yu Kitaev, Alexander Shen, and Mikhail N Vyalyi. Classical and quantum computation, volume 47. American Mathematical Society Providence, 2002.

[2] K. M. Svore, M. B. Hastings, and M. Freedman. Faster Phase Estimation. ArXiv e-prints, April 2013.

[3] Nathan Wiebe and Christopher E Granade. Efficient bayesian phase estimation. arXiv preprint arXiv:1508.00869, 2015.

[4] Cristian Bonato, Machiel S Blok, Hossein T Dinani, Dominic W Berry, Matthew L Markham, Daniel J Twitchen, and Ronald Hanson. Optimized quantum sensing with a single electron spin using real-time adaptive measurements. Nature nanotechnology, 2015.

[5] Brendon L Higgins, Dominic W Berry, Stephen D Bartlett, Howard M Wiseman, and Geoff J Pryde. Entanglement-free heisenberg-limited phase estimation. Nature, 450(7168):393-396, 2007.

[6] Alexander Hentschel and Barry C Sanders. Machine learning for precise quantum measurement. Physical review letters, 104(6):063603, 2010.

[7] Christoph Dankert, Richard Cleve, Joseph Emerson, and Etera Livine. Exact and approximate unitary 2-designs and their application to fidelity estimation. Physical Review A, 80(1):012304, 2009.

[8] Shelby Kimmel, Marcus P da Silva, Colm A Ryan, Blake R Johnson, and Thomas Ohki. Robust extraction of tomographic information via randomized benchmarking. Physical Review X, 4(1):011050, 2014.

[9] Shelby Kimmel, Guang Hao Low, and Theodore J Yoder. Robust calibration of a universal single-qubit gate set via robust phase estimation. Physical Review A, 92(6):062315, 2015.

[10] Easwar Magesan, Jay M Gambetta, and Joseph Emerson. Scalable and robust randomized benchmarking of quantum processes. Physical review letters, 106(18):180504, 2011.

[11] Sarah Sheldon, Lev S. Bishop, Easwar Magesan, Stefan Filipp, Jerry M. Chow, and Jay M. Gambetta. Characterizing errors on qubit operations via iterative randomized benchmarking. Phys. Rev. A, 93:012301, Jan 2016.

[12] Nathan Wiebe, Christopher Granade, and D G Cory. Quantum bootstrapping via compressed quantum hamiltonian learning. New Journal of Physics, 17(2):022005, 2015.

[13] Xavier Boyen and Daphne Koller. Tractable inference for complex stochastic processes. In Proceedings of the Fourteenth conference on Uncertainty in artificial intelligence, pages 33-42. Morgan Kaufmann Publishers Inc., 1998.

[14] Arnaud Doucet, Simon Godsill, and Christophe Andrieu. On sequential monte carlo sampling methods for bayesian filtering. Statistics and computing, 10(3):197-208, 2000.

[15] Jane Liu and Mike West. Combined parameter and state estimation in simulation-based filtering. In Sequential Monte Carlo methods in practice, pages 197-223. Springer, 2001.

[16] Nathan Wiebe, Christopher Granade, Ashish Kapoor, and Krysta M Svore. Bayesian inference via rejection filtering. arXiv preprint arXiv:1511.06458, 2015.

[17] Harald Cramér. Mathematical methods of statistics, volume 9. Princeton university press, 1945.

[18] Karol Zyczkowski and Marek Kus. Random unitary matrices. Journal of Physics A: Mathematical and General, 27(12):4235, 1994.

[19] John Watrous. Theory of quantum information. University of Waterloo Fall, 2011.

[20] Aram W Harrow and Richard A Low. Random quantum circuits are approximate 2-designs. Communications in Mathematical Physics, 291(1):257-302, 2009.

[21] Nathan Wiebe, Christopher Granade, Christopher Ferrie, and D. G. Cory. Hamiltonian learning and certification using quantum resources. Phys. Rev. Lett., 112:190501, May 2014.

[22] Nathan Wiebe, Christopher Granade, Christopher Ferrie, and David Cory. Quantum hamiltonian learning using imperfect quantum resources. Phys. Rev. A, 89:042314, Apr 2014.

[23] Christopher E Granade, Christopher Ferrie, Nathan Wiebe, and D G Cory. Robust online hamiltonian learning. New Journal of Physics, 14(10):103013, 2012.

[24] Markku PV Stenberg, Yuval R Sanders, and Frank K Wilhelm. Efficient estimation of resonant coupling between quantum systems. Physical review letters, 113(21):210404, 2014. 
[25] Steven S Skiena, Warren D Smith, and Paul Lemke. Reconstructing sets from interpoint distances. In Proceedings of the sixth annual symposium on Computational geometry, pages 332-339. ACM, 1990.

[26] Tosio Kato. On the adiabatic theorem of quantum mechanics. Journal of the Physical Society of Japan, 5(6):435-439, 1950.

[27] Donny Cheung, Peter Høyer, and Nathan Wiebe. Improved error bounds for the adiabatic approximation. Journal of Physics A: Mathematical and Theoretical, 44(41):415302, 2011.

[28] Alexander Elgart and George A Hagedorn. A note on the switching adiabatic theorem. Journal of Mathematical Physics, 53(10):102202, 2012.

[29] Daniel A Lidar, Ali T Rezakhani, and Alioscia Hamma. Adiabatic approximation with exponential accuracy for many-body systems and quantum computation. Journal of Mathematical Physics, 50(10):102106, 2009.

[30] AT Rezakhani, AK Pimachev, and DA Lidar. Accuracy versus run time in an adiabatic quantum search. Physical Review A, 82(5):052305, 2010.

[31] Nathan Wiebe and Nathan S Babcock. Improved error-scaling for adiabatic quantum evolutions. New Journal of Physics, 14(1):013024, 2012.

[32] Mária Kieferová and Nathan Wiebe. On the power of coherently controlled quantum adiabatic evolutions. New Journal of Physics, 16(12):123034, 2014.

[33] Gilles Brassard, Peter Hoyer, Michele Mosca, and Alain Tapp. Quantum amplitude amplification and estimation. Contemporary Mathematics, 305:53-74, 2002. 